\title{
Gesundheitsutopien zwischen Fitness und Selbstfindung
}

SIE BRAUCHEN KEINE SCHÖNHEITSOPERATION SIE BRAUCHEN NUR 14 TAGE!

DER ULTIMATIVE

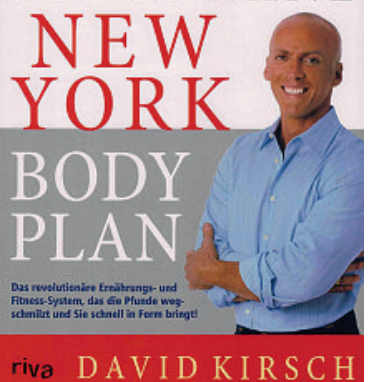

Eine Flut von Gesundheitsratgebern überschwemmt den Buchmarkt mit fliessenden Übergängen in den Schönheitsmarkt. Allen gemeinsam ist, dass Gesundheit als etwas angesehen wird, das man sich selbst erarbeitet.

* Die Literaturangaben finden sich im Internet unter www.saez.ch $\rightarrow$ aktuelle Ausgabe oder $\rightarrow$ Archiv $\rightarrow$ $2010 \rightarrow 16$.

** PD Dr. rer. soc. Eberhard Wolff ist Kulturwissenschaftler, Medizinhistoriker und Mitglied der Redaktion Medizingeschichte der Schweizerischen Ärztezeitung.
Was verstehen wir unter Gesundheit? Oh nein, bitte an diesem Ort keine theoretische Abhandlung! Oft genug ist uns die utopische WHO-Definition von der Gesundheit als Zustand des vollständigen körperlichen, geistigen und sozialen Wohlergehens vor die Nase gehalten worden.

Aber da hat sich mir doch kürzlich eine kleine Erkenntnistür aufgetan und mich ein wenig weiter in die Vorstellungswelt blicken lassen, die Herr und Frau Deutschschweizer von Gesundheit haben. Ich musste eine Zusammenstellung der beliebtesten gesundheitsbezogenen Ratgeberliteratur der letzten Jahre machen. Die seriösen Daten, von Media Control für die Deutschschweizer Verleger und Buchhändler gesammelt, finden wir unter www.hitparade.ch, also gleich neben Martin Suter und Lady Gaga.

Die Bücherhitparade ist das schweizerische Epizentrum einer erstaunlich heterogenen Literaturgattung. In der Spitzengruppe erscheint nur ein einziges klassisches «Hausarzt»-Buch, nämlich das «Hausbuch» von Grönemeyer (ja, das ist der Bruder des Künstlers und der neue Alternativarzt-Superstar) [1] ${ }^{*}$. Das Genre hat sich kaleidoskopartig vervielfältigt. GesundheitsRatgeber gehen fliessend in Sport- oder Kochbücher über. Und sie verstecken sich hinter Formen wie dem Tagebuch oder dem Erfahrungsbericht. Auch Remo Largos Ratgeber-Dauerseller «Babyjahre» [2] ist keine eigentliche Handlungsanleitung.

Was aber haben diese populären Ratgeberbücher mit der utopischen Vorstellung vom vollständigen körperlichen, sozialen und geistigen Wohlergehen zu tun? Mehr, als man denkt! Natürlich zielen sie auf das körperliche Wohlergehen ihrer Leserschaft ab, aber auf welches? Hier und dort geht es um einzelne akute Krankheiten wie beim «Burn-out» - Erfahrungsbericht von Knapp [3]. Doch viele dieser Bestseller schreiben nicht über den hinfälligen, sondern über den gesunden Leib: Wie man ihn «fit» halten kann (im «Ultimativen New York Body Plan» [4]), wie man ihn «vital» macht (mit der «Body Reset»-Methode [5]), und wie man ihn «forever young» erhält (so der Button auf dem «Metabolic Power»-Ratgeber [6]). Den «schlanken» Körper verheissen die Bücher ohnehin, sogar mittels «Schlank im Schlaf» [7]. Fit, vital, jung und schlank: Wenn das keine körperliche Gesundheitsutopie ist - und zwar die ganz aktuelle des Erfolgs- und Leistungskörpers.

Und was ist mit dem sozialen Wohlergehen à la WHO? Auch das kommt nicht zu kurz. «Body Reset» verheisst, dass man mit dieser Methode auch «attraktiv» werde, sogar «ein Leben lang». Und was ist das anderes als ein Faktor sozialen Wohlergehens? Sogar für die medizinischeren Ratgeber gilt dies, etwa wenn Raaflaubs Impotenz-Tagebuch «Tote Hose» im Untertitel heisst: «Worüber Männer schweigen» [8]. Die meisten Ratgeber sehen Gesundheit und Krankheit in der Tat als kommunikativen und damit auch sozialen Prozess, und die Leser danken dies an der Ladentheke.

Einige der Ratgeber-Bestseller wollen den Menschen auch zu einer befriedigenden Sozialbeziehung zu sich selbst verhelfen - wie bei Maja Storchs AntiSchlankheitswahn-Ratgeber «Das Ich-Gewicht» [9]. Auch «Wendepunkte im Lebenslauf» des BestsellerPsychotherapeuten Jürg Willi zielt ganz darauf $a b$, Person und Lebenswelt unter sich verändernden Umständen zu harmonisieren [10]. Ganz zu schweigen von Grönemeyers Anleitung zur «Lebenskunst mit Herz und Seele» [11]. Was ist dies anderes als das Ziel, mit sich in einen Zustand des sozialen Wohlergehens zu kommen?

Und damit haben solche Bücher auch das geistige Wohlergehen im Auge, etwa wenn sie bei der Selbstfindung helfen wollen. Joachim Faulstichs «Das heilende Bewusstsein» geht noch einen Schritt weiter und fragt, wie weit der Geist sogar selbst heilen könne [12].

Wie auch immer man zu den Hauptbotschaften dieser Bücher steht, einen verengten Gesundheitsbegriff kann man diesem heterogenen Genre als Ganzem nicht vorwerfen. Im Gegenteil: Vielleicht hat sich die grosse Gesundheitsutopie der WHO hier besser gehalten als an pragmatischeren Orten, auch wenn sie en détail wohl nicht ganz im Sinne des WHO-Erfinders verstanden wird.

Dafür aber spiegelt das Genre verbreitete Gesundheitsutopien, und dies noch über die WHO-Definition hinaus: Gesundheit als etwas, das man sich selbst erarbeitet, sei es mit Essen, mit Bewegung oder mit Arbeit am Ich; Gesundheit als Praxis, nicht Zustand, ganz im Sinne unserer Gesundheitsgesellschaft; oder Gesundheit als etwas für den Einzelnen hoch Individualisiertes und weniger etwas Hermetisch-Enzyklopädisches, sondern: Jedem sein Special Interest, seine eigene Utopie vom Wohlergehen.

Über unseren Gesundheitsbegriff wird abgestimmt. Nicht nur an der Urne mit dem Stimmzettel, sondern auch in der Buchhandlung mit dem Portemonnaie.

Eberhard Wolff** 


\section{Literatur}

1 Grönemeyer D. Grönemeyers neues Hausbuch der Gesundheit. Reinbek: Rowohlt; 2008.

2 Largo R. Babyjahre. Die frühkindliche Entwicklung aus biologischer Sicht. München: Piper; 2009.

3 Knapp T, Baer N. In den Krallen des Raubvogels. Der Burn-out Erfahrungsbericht. Olten: Textwerkstatt; 2008.

4 Kirsch D. Der ultimative New York Body Plan. München: Riva; 2007.

5 Gehring J. Mit Body Reset attraktiv, schlank, vital, ein Leben lang. Kreuzlingen: Sivita; 2009.

6 Strunz U. Die neue Diät. Fit und schlank durch Metabolic Power. München: Heyne; 2008.

7 Pape D et al. Schlank im Schlaf. München: Gräfe \& Unzer; 2006.
8 Raaflaub W. Tote Hose. Worüber Männer schweigen. Gockhausen: Wörterseh; 2007.

9 Storch M. Mein Ich-Gewicht. Wie das Unbewusste hilft, das richtige Gewicht zu finden. München, Zürich: Pendo; 2008.

10 Willi J. Wendepunkte im Lebenslauf. Persönliche Entwicklung unter veränderten Umständen die ökologische Sicht der Psychotherapie. Stuttgart: Klett-Cotta; 2007.

11 Grönemeyer D. Lebe mit Herz und Seele. Sieben Haltungen zur Lebenskunst. Freiburg/Br.: Herder; 2009.

12 Faulstich J. Das heilende Bewusstsein. Wunder und Hoffnung an den Grenzen der Medizin. München: Knaur; 2006. 